\title{
LE RIPAE DI ROMA IN ETÀ IMPERIALE: QUALCHE EVIDENZA DALLA PIANTA MARMOREA SEVERIANA
}

\section{THE RIPAE OF ROME IN IMPERIAL AGE. SOME EVIDENCE FROM THE SEVERAN MARBLE PLAN}

\author{
MARIA PIA MUZZIOLI \\ maria.pia.muzzioli@uniroma2.it \\ UNIVERSITÁ DEGLI STUDI DI ROMA-TOR VERGATA ${ }^{1}$ \\ [RECIBIDO: 01/03/ 2018; ACEPTADO: 08/04/2018] \\ http://dx.doi.org/10.25267/Riparia.2018.v4.02
}

\section{RÉSUMÉ}

Dans plusieures plaques de marbre du plan sévérien sont représentées les ripae du Tibre à Rome dans l'époque impériale. Les ripae, à droite et à gauche, au sud des grands ponts, apparaissent systématiquement aménagées pour décharger les marchandises provenantes de Portus, les stocker et les distribuer dans la ville. Au nord de l'Insula Tiberina la documentation des fragments est bien limitée. Une différence marquée existe dans l'aménagement de la rive gauche (champ de Mars) et de la rive droite (zone suburbaine, horti). Le prolongement de la délimitation des ripae avec cippi au-delà de la ville, au nord comme au sud, prouve sa fonction de régulation de l'écoulement du Tibre et des activitée liées, non seulement de réglementation urbanistique.

MotS-ClÉ: Forma Urbis Romae Septimii Severi, Tiberis, ripae.

1 Professore associato di Topografia di Roma e dell'Italia Antica. Dipartimento di Storia. Facoltà di Lettere e Filosofia. Edificio B, IV piano, Stanza 11. Via Columbia 1 Roma.

M.P. Muzzioli, "Le ripae di Roma in età imperiale: qualche evidenza dalla pianta marmorea severiana", RIPARIA 4 (2018), 28-45. 
RIPARIA VOL. 4 (2018)

\section{ABstract}

Some tracts of the Tiber ripae in imperial period are depicted in many slabs of the Severan marble plan of Rome. The ripae, right and left, South of the great bridges, appear variously but systematically equiped to discharge goods coming from Portus, to store and distribute them. From the Insula Tiberina to the North the ripae, less documented by the marble fragments, are very differently equiped on the left (Campus Martius) than on the right (suburban area, with horti). The ripae delimitation by cippi further northwards and southwars the urban area prove that the delimitation was evidently functional in controlling the flow of the Tiber and the connected activities, not only the town planning.

KEY WORDS: Forma Urbis Romae Septimii Severi, Tiberis, ripae. 
Nonostante il problema della datazione delle singole rappresentazioni sulla pianta marmorea dell'età di Settimio Severo sia molto aperto, e vada risolto caso per caso $^{2}$, questa forma rimane il principale documento complessivo per una analisi dell'urbanistica di Roma in età imperiale. Molti sono i gruppi di frammenti che riguardano il corso del Tevere, purtroppo durante la loro travagliata vita in epoca moderna - in vari casi tagliati nelle parti senza incisioni.

Un dato comunque sembra sicuro, dal confronto con i frammenti di un'altra pianta marmorea, cosiddetta di via Anicia, e cioè che nella pianta severiana viene tralasciata la linea che si presume indicasse il livello di magra delle acque fluviali, linea che doveva costituire il margine "basso" di quella fascia di terreno chiamata ripa, il cui margine "alto" risulta segnato dai noti cippi ${ }^{4}$.

2 Un riepilogo dei problemi, con bibliografia, in M.P. MuzzioLI, Sui mancati aggiornamenti nella pianta marmorea severiana, in R. COATES-STEPHENS, L. COZZA (edd.), Scritti in onore di Lucos Cozza, 107-122, Roma, 2014. V. anche L. Migliorati, Qualche osservazione sulla forma Urbis severiana, in J. TURChETTO, M. Asolati (edd.), Paesaggi in movimento. Ricerche dedicate a Guido Rosada, 25-40, Padova, 2017.

3 Per i frammenti marmorei fondamentali Pianta marmorea 1960; E. RoDRIGUEZ Almeida, Forma Urbis Marmorea. Aggiomamento generale 1980, Roma, 1980; https:// formaurbis.stanford.edu/. In generale sui problemi relativi al corso del Tevere è ancora utilissimo J. LE GALL, Le Tibre fleuve de Rome dans l'antiquité, Paris, 1953. V. anche J. Le Gall, Il Tevere: fiume di Roma nell'antichità̃ ${ }^{2}$ (C. Mocchegiani Carpano, G. Pisani SARTORIO edd.), Roma, 2005.

${ }^{4}$ La bibliografia sulla lastra di via Anicia è molto ampia, basti qui dare il riferimento alla pubblicazione iniziale (M. Conticello De’ SPagnolis, Il tempio dei Dioscuri nel Circo Flaminio, Roma, 1984.) e ad una importante più recente (E. Rodriguez AlmeIDA, Formae Urbis antiquae. Le mappe marmoree di Roma tra la repubblica e Settimio Severo, Coll. Éc. Fr. Rome 305, Roma, 2002, 43-49). Per quanto riguarda il problema della ripa e delle indicazioni relative in questo documento, dopo un primo inquadramento da parte di Castagnoli (F. CASTAGNOLI, Un nuovo documento per la topografia di Roma antica, StudRom 33, 205-211, 1985 = F. CASTAGNOLI, Topografia antica. Un metodo di studio I. Roma, Roma, 1993, I, 123-130) è importante soprattutto E. Rodriguez AlmeIDA, Formae Urbis antiquae..., 47; v. anche M.P. MuZzIOLI, Le piene del Tevere e la sistemazione delle ripae a Roma: il contributo della Pianta di via Anicia, in E. HermoN (ed.), Société et climats dans l'Empire romain. Pour une perspective historique et systématique de la gestion des ressources en eau dans l'Empire romain, Napoli, 389-407, 2009.

"Le ripae di Roma in età imperiale: qualche evidenza dalla pianta marmorea..." 
Nelle lastre posizionate abbiamo la rappresentazione di una organizzazione urbana complessa e capillare, che dobbiamo attribuire per lo più alle trasformazioni imperiali, con l'enorme sviluppo delle attività commerciali, in particolare legate agli approvvigionamenti via mare, dopo i grandi interventi di Claudio e di Traiano alla foce del Tevere; di tale organizzazione sono testimonianza anche i cippi di delimitazione delle ripae di quegli anni.

Tutta l'area a sud dei grandi ponti venne attrezzata in funzione del carico e scarico di merci e del loro immagazzinamento e distribuzione (tenendo conto ovviamente di tutto l'indotto che queste attività generavano). Gli interventi privati dovettero essere senza dubbio consistenti ma, almeno nelle grandi linee, guidati e controllati dai funzionari imperiali, con l'obiettivo di aumentare al massimo le capacità del sistema.

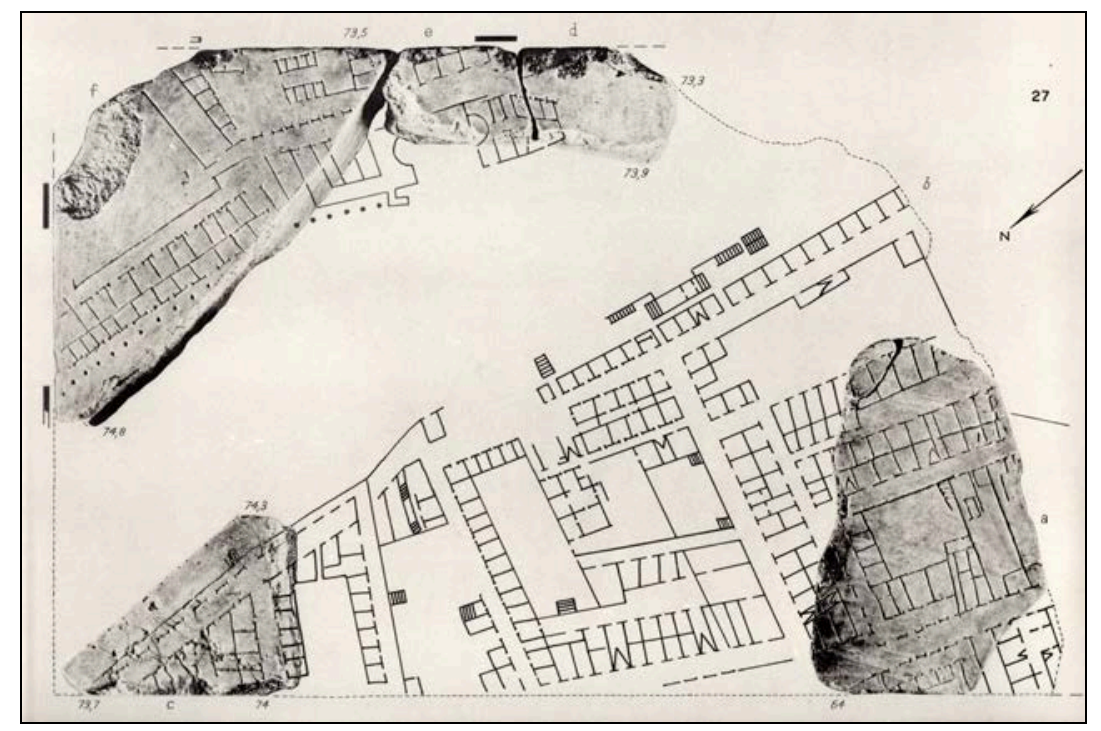

Fig. 1. Lastra 27 (da Pianta marmorea, 1960, Tav. XXVI). 
Se andiamo ad analizzare i frammenti delle lastre 27, 24$28,25^{5}$, cioè il tratto del Tevere a valle del ponte Emilio, il primo elemento che colpisce è la quasi totale occupazione, da parte di strutture di vario genere, di ambedue le sponde del fiume ${ }^{6}$. Solo parti minime rimangono sgombre (ancora con la tradizionale arginatura?).

Nella lastra $27^{7}$ (fig. 1), nella riva sinistra, in successione da sinistra, avanti ad una serie di vani/tabernae, compaiono: una fila di puntini (vi è stato visto un portico affacciato verso il fiume $^{8}$, probabilmente su un elemento viario non delineato che doveva seguirne il corso, oppure elementi per l'ormeggio'), una piccola sporgenza ad L (che Rodriguez Almeida ha interpretato come un piccolo molo ${ }^{10}$ ), una struttura con due esedre (un piccolo bacino? $\left.{ }^{11}\right)$, una limitata successione di ambienti aperti sia verso il fiume che dalla parte opposta. Più oltre si trova uno spazio vuoto, che forse è da ritenere un tratto non edificato. Dall'altra parte del fiume, sempre da sinistra a destra, si può vedere, avanti a vani/tabernae di forma irregolare, un portico a pilastri (con la consueta indicazione a trattini), che potrebbe fare

\footnotetext{
5 Tra la lastra 27 e la 28 sono stati di recente posizionati altri frammenti, in cui si ricostruisce bene la topografia alle spalle, ma manca purtroppo la raffigurazione della riva destra (P.L. TuCCI, Eight fragments of the marble plan of Rome shedding new light on the Transtiberim, PBSR 72, 185-202, 2004, 187-196). E. RodRIGUEZ AlmeIDA, Topografia e vita romana: da Augusto a Costantino, Roma, 2001, 52-57, nota la totale espulsione di edifici da abitazione nelle fasce lungo le ripae nella parte a sud dell'Isola Tiberina, dove si presentano invece più all'interno.

${ }^{6}$ Per un esame di queste aree da una parte e dall'altra del corso fluviale, v. da ultimo E. Rodriguez Almeida, Marziale e Roma. Un poeta e la sua città, Roma, 2014, 521-522 (regione XI), 539-541 (regione XIII), 567-572 (regione XIV).

7 Pianta marmorea 1960, 85-86 (L. CozZA, G. GATTI), Tav. XXVI; E. RodrigueZ AlmeIDA, Forma Urbis Marmorea..., 108, Tav. XIX.

8 Pianta marmorea 1960 , p. 85 (L. CoZZA).

${ }^{9}$ F. Castagnoli, Installazioni portuali a Roma, $M A A R$ 36, 35-42, 1980, $35=\mathrm{F}$. Castagnoli, Topografia antica..., I, 593-595.

${ }^{10}$ E. Rodriguez Almeida, Marziale e Roma...., 521.

${ }^{11}$ F. Castagnoli, Installazioni portuali..., 35 = F. Castagnoli, Topografia antica..., I, 595; E. Rodriguez Almeida, Marįiale e Roma...., 521.
}

"Le ripae di Roma in età imperiale: qualche evidenza dalla pianta marmorea..." 
da pendant al portico sulla riva opposta, ma incluso entro una linea esterna, probabilmente una banchina, allungata anche oltre il portico per un certo tratto, e terminata con un elemento quadrangolare (che si è ipotizzato essere una torre, che potrebbe testimoniare, in rapporto con il piccolo molo sull'altra sponda, l'esistenza di un traghetto ${ }^{12}$ ). Segue uno spazio senza incisioni, poi una fila di ambienti aperti non verso il fiume ma su una strada interna, con una complessa situazione della banchina (di per sé non delineata), con rampe di scale a due livelli ${ }^{13}$, che attestano sia un significativo sbalzo di quota dell'area abitata rispetto al fiume, sia una sistemazione adatta allo scarico anche di merci pesanti e ingombranti. Gli isolati alle spalle, determinati da vie che si collegano abbastanza regolarmente con le ripae, pur con anomalie causate da tracciati in parte precedenti e condizionati dall'orografia della zona, hanno piante riconducibili a grandi impianti per horrea, con spazi aperti, e probabilmente tabernae sulle arterie stradali.

12 E. Rodriguez Almeida, Topografia e vita romana..., 56.

13 Come è stato ben evidenziato dai ritrovamenti (della prima metà del II secolo d.C.) nella zona detta di Pietra Papa, sulla riva destra del fiume (G. JACOPI, Scavi in prossimità del Porto fluviale di San Paolo, MonAL 29, 1943. La pianta è riprodotta da J. LE GALL, Le Tibre fleuve de Rome..., 196-197) le scale e gli anelli d'ormeggio si trovavano all'esterno della delimitazione segnata dai cippi. 


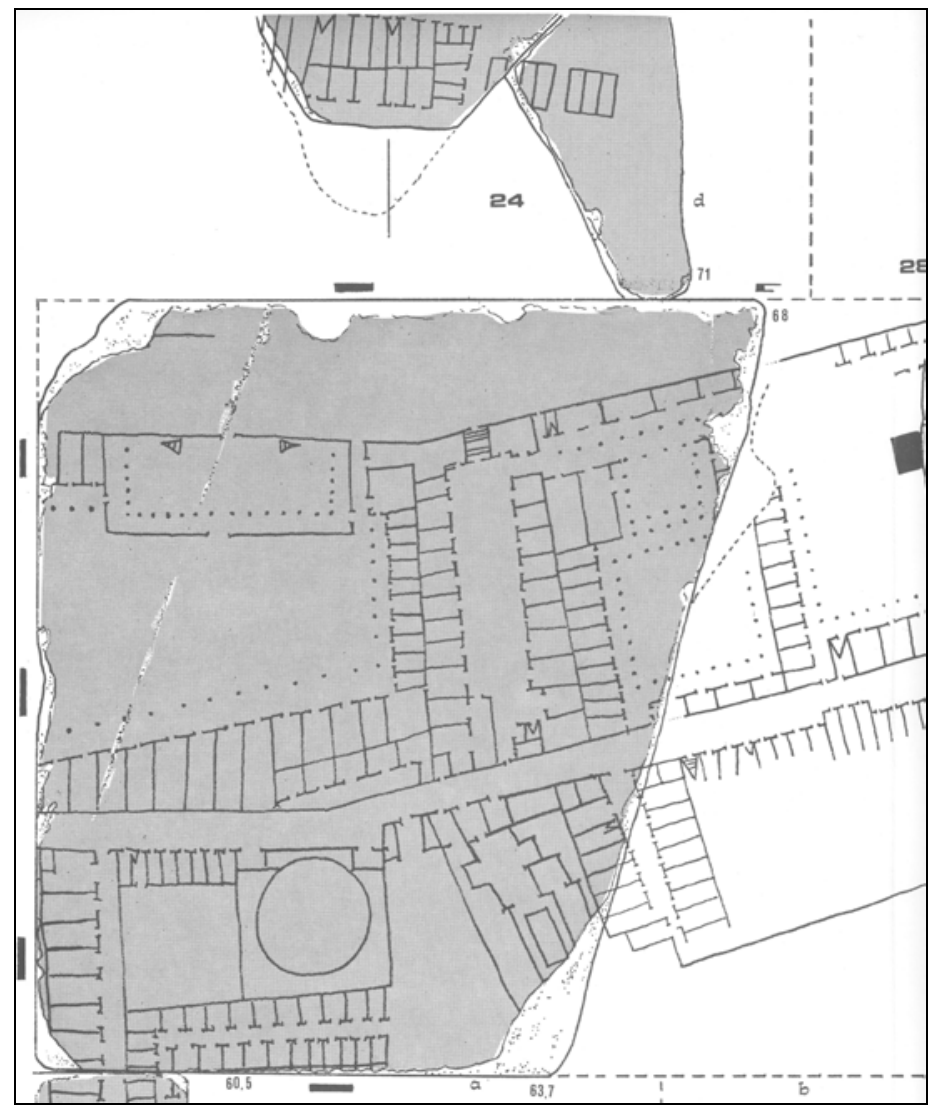

Fig. 2. Lastre 24-28 (da RODRIGUEZ ALMEIDA, 1980, Tav. XX).

Le lastre $24-28^{14}$ (fig. 2) costituiscono un caso unico di rappresentazione congiunta (anche se per un minimo tratto) delle due rive del Tevere. Da esse possiamo anche ricavare una approssimata larghezza del fiume in questo tratto: circa $65 \mathrm{~m}$. Nella lastra 24 abbiamo serie di ambienti e tra essi passaggi diretti al fiume; la ripa non è maggiormente indicata. Nella lastra 28

14 Pianta marmorea 1960, 81-82 (G. GATTi), Tav. XXIV; 87-88 (G. CARETTONI), Tav. XXVII; E. RodrigueZ AlmeIDA, Forma Urbis Marmorea...., 102-105, Tav. XVI (con prosecuzione a Tav. XX), fig. 24; 108, Tav. XX.

"Le ripae di Roma in età imperiale: qualche evidenza dalla pianta marmorea..." 
invece una linea continua ${ }^{15}$, interrotta in pochi punti (in un caso con una scala), deve indicare una banchina, o perlomeno una delimitazione artificiale (sulla sinistra in un'ampia area porticata con accesso diretto dal fiume è stato visto uno scalo interno ${ }^{16}$ ). Alle spalle vasti complessi edilizi interpretabili come horrea.

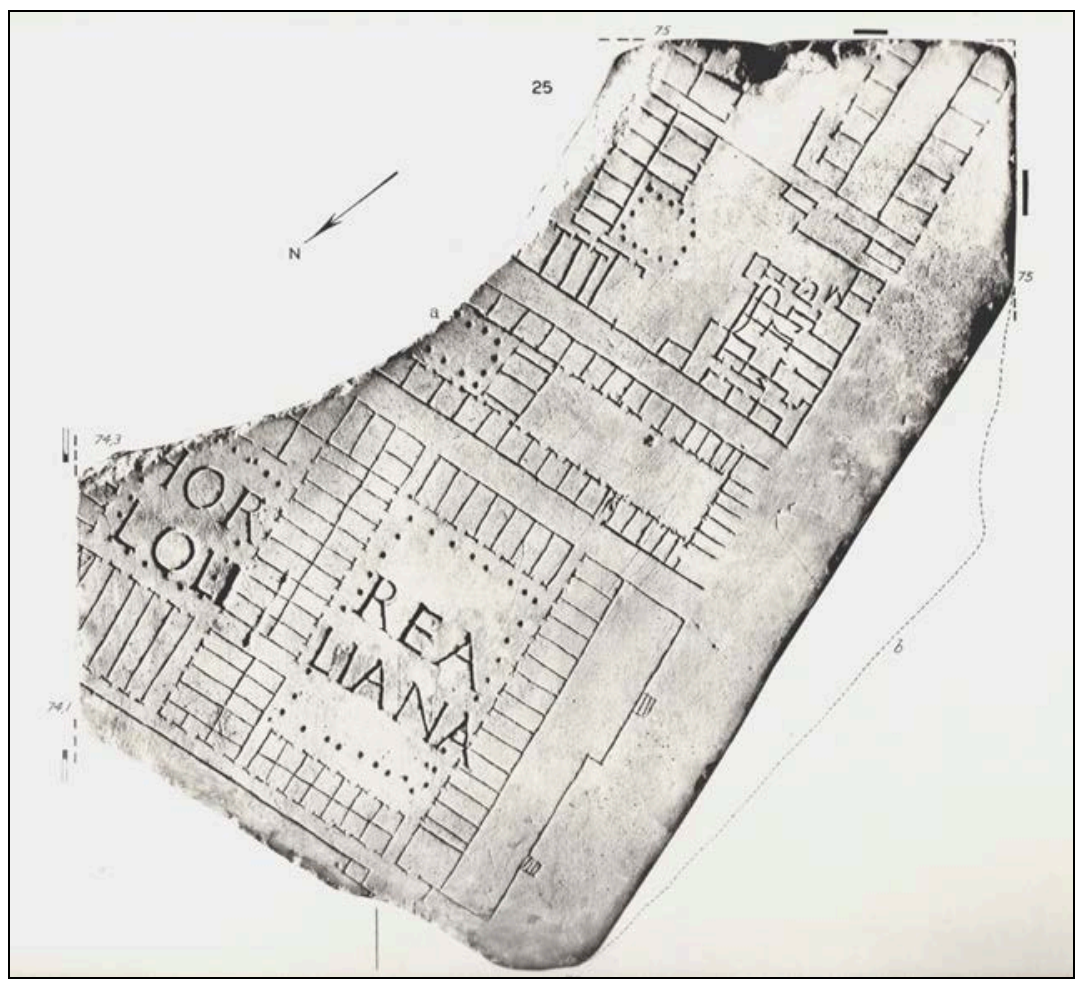

Fig. 3. Lastra 25 (da Pianta marmorea, 1960, Tav. XXV).

\footnotetext{
15 Secondo E. Rodriguez Almeida, Topografia e vita romana..., 55, si tratta della linea dell'acqua alta, e non sarebbero rappresentati i moli veri e propri.

16 Pianta marmorea 1960, 87 (G. CARETTONI). 
Nel tratto più a valle (lastra $25^{17}$ : fig. 3 ), sulla riva sinistra, gli horrea Lolliana hanno su un lato una larga banchina, con due scale di accesso. La lastra marmorea è stata qui ritagliata sia eliminando una parte del corso d'acqua sia, in perpendicolare, un'area forse vuota delimitata da una linea incisa. Oltre agli horrea Lolliana le altre strutture presenti, che sembrano avere le stesse funzioni, hanno però soluzioni diverse nell'affaccio sul fiume: dei vani aperti (o forse qui si può ipotizzare una dimenticanza dell'incisore?), dei muri chiusi in corrispondenza di quello che è stato riconosciuto come un edificio termale, e infine una serie di vani con aperture verso il fiume. Come è stato notato ${ }^{18}$, con la costruzione delle mura Aureliane, che chiusero questo tratto di sponda senza aperture, la funzionalità di queste attrezzature dovette cessare.

Il quadro che si ricava da queste raffigurazioni è quello di una sistemazione artificiale complessiva che, per lo più con banchine, segnava il limite della ripa. Più a monte, varie differenze si possono riscontare nel tratto della ripa sinistra di fianco all'Isola Tiberina (lastra 32, fig. 4), dove possiamo avere anche il supporto della pianta di via Anicia. Per quanto riguarda la pianta severiana un nuovo frammento ${ }^{19}$ si è aggiunto recentemente agli altri, completando l'iscrizione circus Flaminius e mostrando una prosecuzione dell'assetto già noto (con portici e vani aperti sia verso il fiume che verso le aree più interne (in primis il circo Flaminio) collegandosi all'area del teatro di Marcello, che con la sua costruzione raggiungeva praticamente il bordo del fiume.

17 Pianta marmorea 1960, 83-84 (G. GatTi), Tav. XXV; E. RodrigueZ AlmeidA, Forma Urbis Marmorea..., 106, Tav. XVIII, fig. 25.

${ }^{18} \mathrm{~F}$. DE CAPRARIIS, I porti della città nel IV e V secolo d.C., in The transformations of Vrbs Roma in late Antiquity, JRA, Suppl. S., 33, 216-234, 1999, 219; F. DE CAPRARIIS, I porti urbani tra continuità e trasformazioni, in Puertos Fluviales Antiguos: Ciudad, Desarrollo e Infraestructuras, Valencia, 261-275, 2001, 264-265.

19 G. Filippi, P. Liverani, Un nuovo frammento della Forma Urbis con il Circus Flaminius, RPAA 87, 69-88, 2014-2015.

"Le ripae di Roma in età imperiale: qualche evidenza dalla pianta marmorea..." 
RIPARIA VOL. 4 (2018)

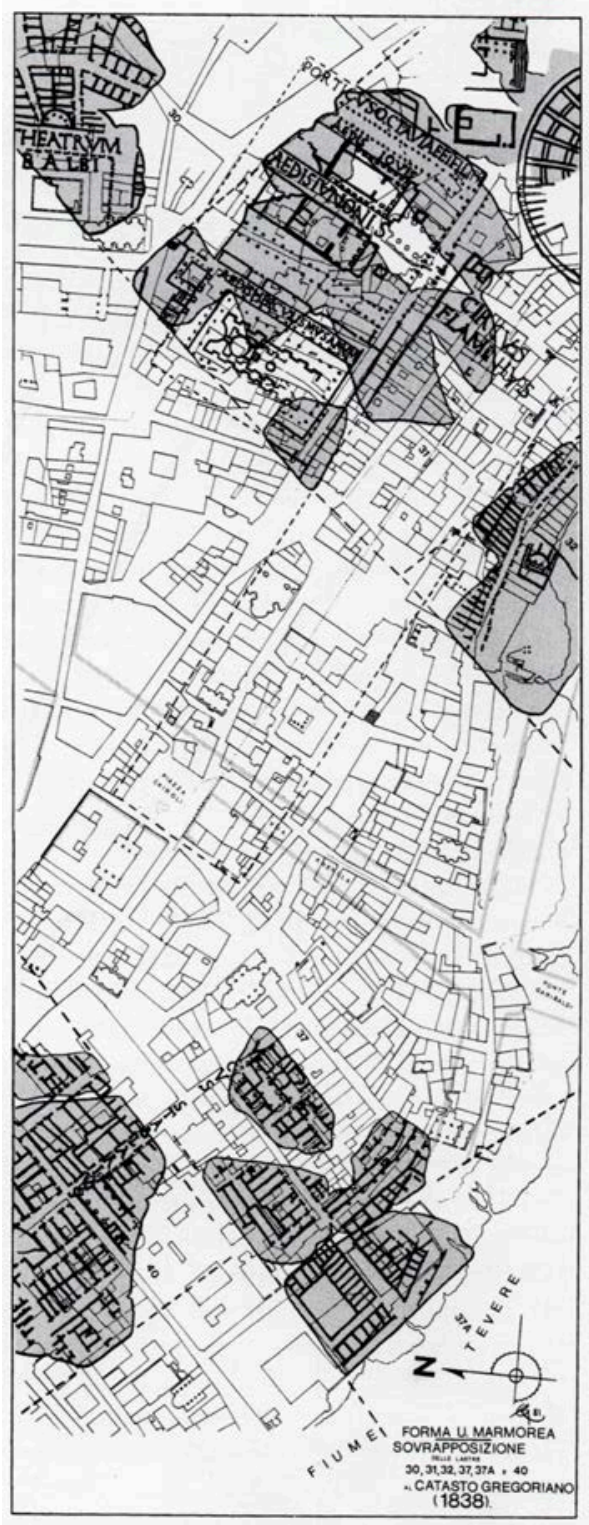

Fig. 4. Pianta con posizionamento delle lastre 32, 37, 37 A (da RODRIGUEZ ALMEIDA 1983, fig. 3). 
Una situazione con alcune analogie, ma anche con differenze significative, possiamo riscontrare nel tratto del Tevere a nord dell'Isola Tiberina, nella grande ansa che includeva il Campo Marzio in senso lato, zona che, dal momento in cui furono costruiti i grandi ponti in pietra (ponte Emilio, ponte Fabricio, Ponte Cestio, ponte di Agrippa) non poteva essere raggiunta da grosse imbarcazioni provenienti dalla foce. Il settore più meridionale dell'ansa - una fascia limitata ai tempi di Claudio, poi una fascia maggiore con Vespasiano (ribadita da Adriano) venne a quel punto lasciato al di fuori del Campo Marzio vero e proprio, soggetto a restrizioni di carattere giuridico, allargando l'area pomeriale oltre le mura repubblicane, con il risultato di una veloce urbanizzazione, che, oltre che archeologicamente, è ben attestata da gruppi di frammenti della pianta marmorea.

In modo particolare ci interessa il frammento 602 (anch'esso con un lato tagliato artificialmente per eliminare spazi bianchi), posizionato da Rodriguez Almeida nella lastra definita $37 \mathrm{~A}^{20}$ (nella topografia attuale tra via delle Zoccolette e il Lungotevere dei Vallati) (fig. 4): il fiume è delimitato da un portico con pilastri o colonne fino ad una strada perpendicolare, al di là della quale è una struttura a vani chiusi (edificati, secondo Rodriguez Almeida, sul pendio fluviale); alle spalle una serie di isolati di non grandi dimensioni e con vani paralleli in un tessuto fitto con una viabilità abbastanza regolare.

\footnotetext{
20 E. Rodriguez Almeida, Forma Urbis Marmorea..., 136-139; E. Rodriguez ALMEIDA, Un nuovo frammento della Forma Urbis Marmorea, in Città e architettura nella Roma imperiale, ARID, Suppl. X, 87-92, 1983, figg. 1, 2 e 3; E. Rodriguez ALmEIDA, Topografia e vita romana..., 57-58. Per l'inquadramento nel tessuto viario v. L. QuiLICI, Il Campo Marzio occidentale, in Città e architettura nella Roma imperiale, ARID, Suppl. X, 5985,1983
}

"Le ripae di Roma in età imperiale: qualche evidenza dalla pianta marmorea..." 
RIPARIA VOL. 4 (2018)

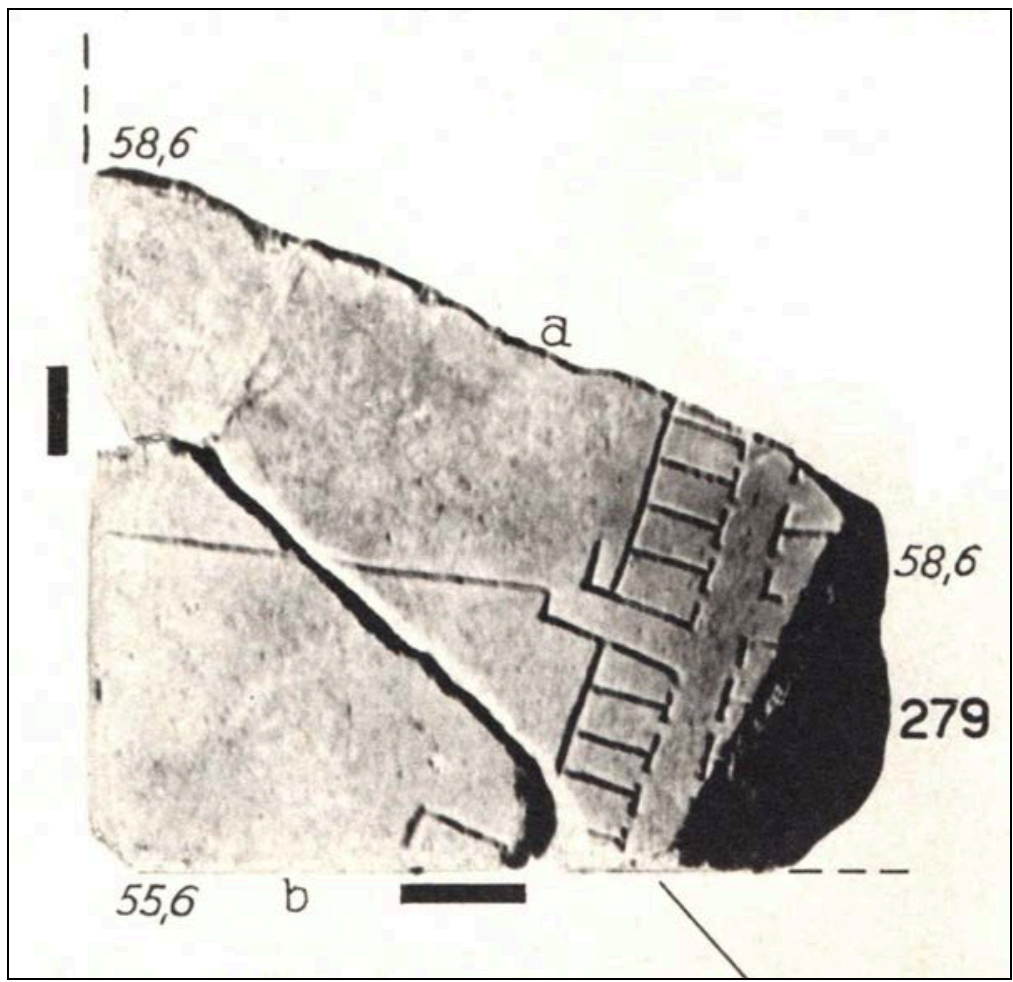

Fig. 5. Frammento 279 (da Pianta marmorea, 1960, Tav. XLIV).

Un dato forse da recuperare è inoltre la proposta affacciata da Rodriguez Almeida a proposito del frammento 279 (fig. 5), posizionato dubitativamente sulla riva destra del fiume (via della Lungara, tra le vie D'Alibert e delle Mantellate). Sarebbe da riconoscervi un piccolo molo e, nella linea retta rappresentata, un'indicazione di un traghetto "a filo", cioè con una barca collegata a un cavo teso tra una riva e l'altra ${ }^{21}$. Ci troveremmo

${ }^{21}$ E. Rodriguez Almeida, Forma Urbis Marmorea, nuovi elementi di analisi e nuove ipotesi di lavoro, MEFRA 89,1, 219-256, 1977, 247-248; cfr. E. RoDRIGUEZ ALMEIDA, Forma Urbis Marmorea..., 159. Un altro molo molto noto, sporgente obliquo sul corso del fiume, cui si sovrappose un tempietto in epoca successiva, è quello di Tor di Nona (oltre alla prima descrizione di D. MARCHETTI, Di un antico molo per lo sbarco dei marmi riconosciuto sulla riva sinistra del Tevere, $B C A R, 45-60$, 1891, vedi G. CRESSEDI, 
subito a nord del complesso della "villa della Farnesina", dove anche in età moderna è documentato un traghetto di questo genere. Segnalo che dalla parte opposta del fiume, nell'area del Campo Marzio, sono stati di recente rinvenuti due cippi di delimitazione delle ripae, di cui uno in situ. Si tratta di un nuovo cippo flavio, del 72 d.C. Esso, infisso in un piano a quota 9,55 s.l.m., delimitava un'area fino a quel momento con una limitata presenza di edifici, ma presto venne interrato per un rialzamento di livello e inglobato, rimanendo al suo posto, in strutture diverse, attribuite a utilizzazioni forse commerciali o artigianali, datate nel II secolo d.C. ${ }^{22}$, in una situazione che si configura abbastanza simile a quella riscontrata un poco più a valle nelle lastre 37, 37A. È qui evidente che la linea definita dal cippo venne superata, non sappiamo se abusivamente o con operazioni controllate (il rialzamento di livello deve far parte di un intervento più generale) e con successivi ripristini. È significativo che su questa sponda nella parte settentrionale fossero presenti più scali, di servizio per i rifornimenti che arrivavano a Roma via Tevere da nord (prodotti agricoli, materiali da costruzione, laterizi e ceramiche), scali di particolare importanza, in cui il controllo pubblico doveva essere ben presente se, quando vennero costruite le mura Aureliane, in corrispondenza di essi vennero aperte delle posterulae ${ }^{23}$.

I porti fluviali in Roma antica, $R P A A$ 25-26, 53-65, 1949-51, 62-65; F. CASTAGnOLI, Installazioni portuali..., 35; E. LA ROCCA, La riva a mezzaluna. Culti, agoni, monumenti funerari nel Campo Marzio occidentale, Roma, 1984, 62-65; F. DE CAPRARIIS, I porti della città..., 220; F. DE CAPRARIIS, I porti urbani..., 266). Non ne abbiamo però purtroppo la corrispondente documentazione nei frammenti severiani.

${ }^{22}$ F. FILIPPI, Il quartiere dello stabulum Factionis Russatae nel Campo Marzio occidentale. Rapporto preliminare sull'indagine di scavo di largo Perosi (2009-2014), in F. FILIPPI (ed.), Campo Marzio. Nuove ricerche (Atti del Seminario di Studi sul Campo Marzio, Roma 18-19 marzo 2013), Roma, 393-442, 2015, 419-423, 433; G.L. GREGORI, Documenti epigrafici dal contesto di largo Perosi in Campo Marzio: due nuovi termini del Tevere e altri reperti, in F. Filippi (ed.), Campo Marzio. Nuove ricerche (Atti del Seminario di Studi sul Campo Marzio, Roma 18-19 marzo 2013), Roma, 443-451, 2015, 443-445.

23 Cfr. a questo proposito de F. DE CAPRARIIS, I porti della città..., 220; F. DE CAPRARIIS, I porti urbani..., 266.

"Le ripae di Roma in età imperiale: qualche evidenza dalla pianta marmorea..." 
È stato più volte notato come la situazione della riva destra di fronte al Campo Marzio, per cui non abbiamo purtroppo a disposizione frammenti della pianta marmorea posizionati, abbia avuto uno sviluppo particolare, per la presenza di complessi privati (horti), esterni all'area urbana, anche di notevole prestigio (in primo luogo la "villa della Farnesina"), specialmente tra la fine della repubblica e il primo secolo dell'impero, appartenenti in qualche caso a membri della famiglia imperiale, con strutture edilizie che si affacciavano direttamente sul fiume, e che condizionarono fortemente il tracciato della linea di ripa. E' stata qui analizzata con particolare cura, fin dalla prima scoperta $^{24}$, e di recente riesaminata ${ }^{25}$, la dislocazione dei cippi di delimitazione del Tevere, eccezionalmente numerosi e disposti con andamento tutt'altro che regolare, e non riprendo quindi qui il discorso. Meno attenzione è stata invece riservata alla restante situazione archeologica, anch'essa nota prevalentemente per le scoperte avvenute durante le grandi distruzioni causate dalla costruzione dei muraglioni del Lungotevere. Alcuni dati recuperati sono invece interessanti perché, oltre all'individuazione di un notevole numero di strutture avanzate sulla riva del fiume, permettono la ricostruzione di un sistema di scolo a protezione di un argine a una certa distanza dalla riva ${ }^{26}$, lungo cui poteva almeno in alcuni tratti correre una via di alaggio ${ }^{27}$. La presenza dell'argine, che purtroppo non possiamo datare e di cui non riusciamo neanche a capire bene il rapporto cronologico con le strutture private (di buon livello costruttivo) che raggiungevano

24 D. Marchetti, Prati di Castello, NSA, 82-88, 1890, soprattutto 86; CH. Hülsen, Der Tiber und die Brücken, $M D A I(R)$ 6, 130-150, 1891; CIL VI, pp. 3109-3110; cfr. J. LE GALL, Le Tibre fleuve de Rome..., 149-166.

25 A. LONARDI, La cura riparum et alvei Tiberis. Storiografia, prosopografia e fonti epigrafiche, BAR Int. Ser., 2464, Oxford, 2013, soprattutto 39-43.

26 M.P. MuZzioli, La riva destra del Tevere tra i ponti Cavour e Margherita, in A. SERrA (ed.), Humanitas. Studi per Patrizia Serafin, 163-174, Roma, 2015, 167-170, con bibliografia precedente.

${ }^{27}$ L. QUiLICI, Il Tevere e l'Aniene come vie d'acqua a monte di Roma in età imperiale, in Il Tevere e le altre vie d'acqua del Lazio antico, (Archeologia Laziale VII,2), 198-217, 1986, 202. 
l'acqua, doveva avere come principale funzione quella di difendere la pianura retrostante (attuali Prati), e in un certo senso ci permette di intuire come l'organizzazione dei terreni al bordo del fiume fosse complessa e sviluppata in più fasi, e vada quindi ricostruita caso per caso. Quello che sembra potersi escludere è che qui si presentassero delle banchine continue.

Resta comunque il fatto che l'estensione stessa della delimitazione con cippi - a partire da quella tardo repubblicana, fino ad arrivare ai restauri più tardi - andava ben al di là di quella che poteva essere considerata area urbana (almeno da Ponte Milvio fino a Pian Due Torri a valle di Pietra $\mathrm{Papa}^{28}$, a meno che non si voglia pensare che arrivasse fino ad Ostia, dove sono stati trovati cippi, ma forse di una delimitazione a parte ${ }^{29}$. Ciò permette di legare la sistemazione delle ripae non soltanto alle esigenze della vita cittadina, ma anche alla funzionalità del regolare andamento del corso fluviale e delle più specifiche attività connesse, in una interazione continua, e differenziata nel tempo, tra gestione dei fenomeni naturali e sviluppi urbanistici.

Certamente la documentazione delle immagini della pianta marmorea severiana non può chiarirci alcuni problemi, come il rapporto pubblico-privato, la presenza di abusi rispetto alla legislazione vigente, o in alcuni casi le eccezioni entro quella stessa legislazione, tuttavia consente di cogliere, con una immediatezza visiva molto superiore rispetto ad altre fonti, la grande varietà di situazioni, frutto di interventi cronologicamente diversi e di diverse necessità. Non ultimo motivo di interesse sono i dubbi che ci pongono queste immagini della realtà, giunte a noi senza intermediari dall'antichità, e che ci spingono ad approfondire la ricerca, al di là di soluzioni schematiche e rigide.

\footnotetext{
${ }^{28}$ Per il quadro archeologico v. F. CASTAGNOLI, Installazioni portuali...

29 J. LE GALL, Le Tibre flewve de Rome..., 138, 156-157, 163; A. LONARDi, La cura riparum..., 44; v. anche E. Hermon, La liberté de navigation et la délimitation des rives publiques à Ostie au temps de Caninius, in I. PIRO (ed.), Scritti per Alessandro Corbino, 3, Tricase (LE), 601-622, 2016.
}

"Le ripae di Roma in età imperiale: qualche evidenza dalla pianta marmorea..." 


\section{Bibliografia}

F. CASTAGNOLI, Installazioni portuali a Roma, $M A A R$ 36, 35-42, 1980.

F. CASTAgnOLI, Un nuovo documento per la topografia di Roma antica, StudRom 33, 205-211, 1985.

F. Castagnoli, Topografia antica. Un metodo di studio I. Roma, Roma, 1993.

M. Conticello De’ SPAGnOlis, Il tempio dei Dioscuri nel Circo Flaminio, Roma, 1984.

G. CREssedi, I porti fluviali in Roma antica, $R P A A$ 25-26, 53-65, 1949-51.

F. DE CAPRARIIS, I porti della città nel IV e V secolo d.C., in The transformations of Vrbs Roma in late Antiquity, JRA, Suppl. S., 33, 216-234, 1999.

F. DE CAPRARIIS, I porti urbani tra continuità e trasformazioni, in Puertos Fluviales Antiguos: Ciudad, Desarrollo e Infraestructuras, Valencia, 261275, 2001.

G. FILIPPI, P. LIVERANI, Un nuovo frammento della Forma Urbis con il Circus Flaminius, RPAA 87, 69-88, 2014-2015.

F. FILIPPI, Il quartiere dello stabulum Factionis Russatae nel Campo Marzio occidentale. Rapporto preliminare sull'indagine di scavo di largo Perosi (2009-2014), in F. FILIPPI (ed.), Campo Marzio. Nuove ricerche (Atti del Seminario di Studi sul Campo Marzio, Roma 18-19 marzo 2013), Roma, 393-442, 2015.

G.L. Gregori, Documenti epigrafici dal contesto di largo Perosi in Campo Marzio: due nuovi termini del Tevere e altri reperti, in F. FILIPPI (ed.), Campo Marrio. Nuove ricerche (Atti del Seminario di Studi sul Campo Marzio, Roma 18-19 marzo 2013), Roma, 443-451, 2015.

E. Hermon, La liberté de navigation et la délimitation des rives publiques à Ostie au temps de Caninius, in I. PIRO (ed.), Scritti per Alessandro Corbino, 3, Tricase (LE), 601-622, 2016.

CH. HÜLSEN, Der Tiber und die Brücken, MDAI(R) 6, 130-150, 1891.

G. JACOPI, Scavi in prossimità del Porto fluviale di San Paolo, MonAL 29, 1943.

E. LA RoCCA, La riva a mezzaluna. Culti, agoni, monumenti funerari nel Campo Marzio occidentale, Roma, 1984.

J. LE GALL, Le Tibre fleuve de Rome dans l'antiquité, Paris, 1953. 
J. LE GALL, Il Tevere: fiume di Roma nell'antichitàn ${ }^{2}$ (C. MOCCHEGIANI CARPANO, G. Pisani SARTORIO edd.), Roma, 2005.

A. LONARDI, La cura riparum et alvei Tiberis. Storiografia, prosopografia e fonti epigrafiche, BAR Int. Ser., 2464, Oxford, 2013.

D. MARCHETTI, Prati di Castello, NSA, 82-88, 1890.

D. MARCHETTI, Di un antico molo per lo sbarco dei marmi riconosciuto sulla riva sinistra del Tevere, BCAR, 45-60, 1891.

L. MigliorATI, Qualche osservazione sulla forma Urbis severiana, in J. Turchetto, M. Asolati (edd.), Paesaggi in movimento. Ricerche dedicate a Guido Rosada, 25-40, Padova, 2017.

M.P. MuZzioli, Le piene del Tevere e la sistemazione delle ripae a Roma: il contributo della Pianta di via Anicia, in E. HERMON (ed.), Société et climats dans l'Empire romain. Pour une perspective historique et systématique de la gestion des ressources en eau dans l'Empire romain, Napoli, 389-407, 2009.

M.P. MUZZIOLI, Sui mancati aggiornamenti nella pianta marmorea severiana, in R. COATES-STEPHENS, L. COZZA (edd.), Scritti in onore di Lucos Cozza, 107-122, Roma, 2014.

M.P. MuZzIOLI, La riva destra del Tevere tra i ponti Cavour e Margherita, in A. SERRA (ed.), Humanitas. Studi per Patrizia Serafin, 163174, Roma, 2015.

G. CARETTONI, A.M. COLINI, L. COZZA, G. GATTI, La pianta marmorea di Roma antica (Forma urbis Romae), Roma 1960.

L. QUILICI, Il Campo Marzio occidentale, in Città e architettura nella Roma imperiale, ARID, Suppl. X, 59-85, 1983.

L. QUILICI, Il Tevere e l'Aniene come vie d'acqua a monte di Roma in età imperiale, in Il Tevere e le altre vie d'acqua del Lazio antico, (Archeologia Laziale VII,2), 198-217, 1986.

E. Rodriguez AlmeidA, Forma Urbis Marmorea, nuovi elementi di analisi e nuove ipotesi di lavoro, MEFRA 89,1, 219-256, 1977.

E. Rodriguez AlmeidA, Forma Urbis Marmorea. Aggiornamento generale 1980, Roma, 1980.

E. Rodriguez ALmeidA, Un nuovo frammento della Forma Urbis Marmorea, in Città e architettura nella Roma imperiale, ARID, Suppl. X, 8792, 1983.

E. Rodriguez ALmeIDA, Topografia e vita romana: da Augusto a Costantino, Roma, 2001.

"Le ripae di Roma in età imperiale: qualche evidenza dalla pianta marmorea..." 
RIPARIA VOL. 4 (2018)

E. RoDRIGUEZ ALMEIDA, Formae Urbis antiquae. Le mappe marmoree di Roma tra la repubblica e Settimio Severo (Coll. Éc.Fr. Rome 305), Roma, 2002.

E. Rodriguez AlmeIDA, Marziale e Roma. Un poeta e la sua città, Roma, 2014.

P.L. TUCCI, Eight fragments of the marble plan of Rome shedding new light on the Transtiberim, PBSR 72, 185-202, 2004. 